\title{
Matching Fabric Based on Wavelet Kernel Signature (WKS) and Drape Indicators *
}

\author{
Zhicai $\mathrm{Yu}^{\mathrm{a}}$, Yueqi Zhong ${ }^{\mathrm{a}, \mathrm{b}, *}, \quad$ Haoyang $\mathrm{Xie}^{\mathrm{a}}$ \\ ${ }^{a}$ College of Textiles, Donghua University, Shanghai 201620, China \\ ${ }^{\mathrm{b}}$ Key Laboratory of Textile Science \& Technology of Ministry of Education, College of Textiles, \\ Donghua University, Shanghai 201620, China
}

\begin{abstract}
The drape performance of the fabric is an important part of the fabric style, as well as a special expression of the structural parameters and mechanical properties. The comprehensive drape performance of fabric can support fabric matching. This study was conducted to match fabric based on 3D triangular meshes of draped fabrics. Firstly, the three-dimensional (3D) point cloud of draped fabric was scanned with a self-built 3D scanning device followed with triangulation. Secondly, three drape indicators, i.e., drape coefficient, the number of fabric drape nodes, and the solid rate of a draped fabric was extracted, as well as the wavelet kernel signatures (WKS) of triangular meshes. The WKS of vertices on the 3D boundary was selected and clustered into different classes with K-Means. With the cluster centers, the WKS of vertices on the boundary was encoded into a vector with a fixed length. Finally, two methods for matching fabrics were proposed based on WKS+DR and a vector consisting of three drape indicators separately. The results show that WKS $+\mathrm{DR}$ outperforms the vector consisting of drape indicators in characterizing the drape configuration details of a draped fabric. The accuracy of the method based on WKS+DR could reach $89.6 \%$. The fabrics obtained with this method are close to the matching fabrics in both drape-ability and details of a triangle surface.
\end{abstract}

Keywords: Fabric Matching; Draped Fabric; Triangular Mesh; Wavelet Kernel Signature (WKS)

\section{Introduction}

The drape performance of the fabric is an important part of the fabric style. In one aspect, drape performance depends on the mechanical properties, structural parameters of fabric as well as the environmental factors [1]. In another aspect, drape performance is also an expression of the structural parameters and mechanical properties of fabrics [2]. That is, drape performance could be though as an implicit function of fabric parameters. Therefore, drape performance could provide significant guidance for fabric comparison and matching.

*Project supported by the National Natural Science Foundation of China (Grant No. 61572124) and the Special Excellent Ph.D. International Visit Program by Donghua University (DHU).

* Corresponding author.

Email address: zhyq@dhu.edu.cn (Yueqi Zhong). 
The research object of fabric drape may be a two-dimensional top-view image [3] or a threedimensional point cloud model. A two-dimensional image of a draped fabric is easy to obtaining and analyzing, while 3D models contain more information than two-dimensional images. With the development of 3D scanning technology, the cost of 3D modeling of draped fabrics has been significantly reduced, which results in the rapid development of studies about 3D models of draped fabrics. However, so far, most of the research on the three-dimensional modeling of the draped fabrics have been focusing on the acquisition of 3D point clouds or triangular meshes of the draped fabrics. For example, Wang et al [4] identified the contour line information in the panoramic raster image, located the grating stripe, and obtained the height of the space where the grating stripe is located. With this method, a three-dimensional image of the specimen's draping morphology is constructed with a bi-cubic Bezier surface. Mah et al [5] captured 3D images of fabric drape through a 3D body scanning and studied the distribution of air gaps between the draped fabric and the outside edge of a cylinder. Hu et al [6] proposed a simple effective method that can reconstruct and measure the fabric drapes with a smartphone. The breakthrough of their study lies in the simple approach of obtaining the 3D point cloud of fabric drape. These reports mentioned above show that there is little research on the comparison of comprehensive drape performance. The reason lies that fabric performance contains not only the drape ability but also the drape configurations. Besides, the result of the fabric drape is discrete. Niwa et al [7] tested 145 fabrics for the female dress and 138 fabrics for the male shirt. The result revealed that the same fabric could possess different DRs under the same experimental condition and different drape times. For the variance of fabric drape, Kenkare et al [8] pointed out that fabric drape is dependent on structure parameters, mechanical properties, and the environment. Besides, these factors were depended on other factors. Therefore, fabrics do not fall in the same con?guration each time they drape.

However, there is a factor that we cannot ignore. The structure of the fabric determines the properties of the fabric. Although there are discrete drape results for the same fabric under the same experiment condition, the local deformation of a draped fabric may be stable. Therefore, we can infer the fabric performance with the local deformation. How to describe the local deformation of a triangular mesh? Inspired by the corresponding and analysis of 3D shapes, we proposed to analyze the 3D triangular mesh of a draped fabric with a wavelet kernel signature (WKS). WKS is a three-dimensional shape descriptor. It performs great in distinguishing 3D shapes. Mathieu Aubry et al [9] have experimentally proved that WKS is more effective in analyzing 3D shapes than other types of 3D shape descriptors, such as Fourier descriptors (FD), 3D Rotation Invariant Local Binary (3D LBP), 3D scale-invariant feature transform feature vector (3D SIFT) and heat kernel signature (HKS).

In this study, we obtain the 3D point cloud of draped fabrics followed with surface reconstruction. Then the WKS of a draped fabric is calculated and encoded into a vector. A new method was proposed to match fabrics, i.e., matching fabric with the encoded WKS as well as DR. In the comparison experiment, a vector consisting of three drape indicators was used to match fabrics. At last, the two methods are compared and analyzed [10].

\section{Material and Methods}

The pipeline of matching fabric based on WKS+DR is shown in Fig. 1.

As shown in Fig. 1, the step of "fabric scanning" refers to obtaining the 3D point cloud of a 


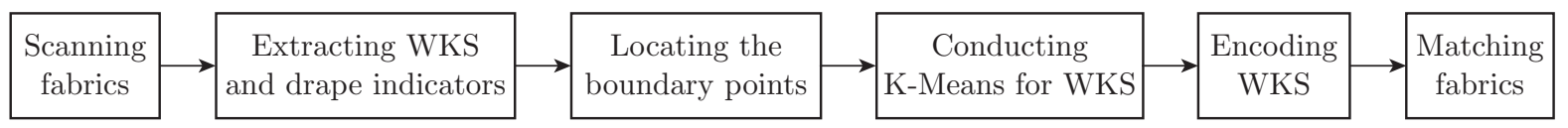

Fig. 1: The pipeline of fabric matching based on WKS+DR

draped fabric via a self-built scanning device. The step of "extracting WKS and drape indicators" refers to extracting the WKSs of vertices in the whole triangular mesh, as well as some drape indicators. The step of "locating the boundary points" refers to figure out which vertex is on the boundary of a draped fabric. The step of "conducting K-Means" refers to cluster the WKS of boundary vertices with K-Means. The step of "encoding WKS" refers to statistics of the clustering of WKS of boundary vertices of each draped fabric. The step of "fabric matching" refers to evaluate the Euclidean distance between the encoded WKSs of matching fabrics and given fabrics.

\subsection{Preparing Fabric Samples}

Fifty-one fabrics collected from the market were washed and ironed to remove wrinkles on the surface of the fabric. The fabric samples were cut into circular specimens with a radius of 120 $\mathrm{mm}$. The centers of the specimens were located and marked. The fabric was conditioned in a constant temperature and humidity laboratory (temperature: $23 \pm 2^{\circ} \mathrm{C}$, humidity: $65 \% \pm 2 \%$ ) for 48 hours.

\subsection{The Constructing and Calibration of the Scanning Device}

The scanning device for a draped fabric is constructed with four depth cameras by referring to the method of $\mathrm{Wu}$ et al [11]. The scanning device is shown in Fig. 2(b).

The circular specimens were centered on a supporting disk with a radius of $60 \mathrm{~mm}$ surrounded by four RGB-Depth cameras, as shown in Fig. 2(b). The angle between two adjacent cameras is $90^{\circ}$. To reach an accurate scanning result, a T-shaped checkerboard as shown in Fig. 2(b) was used to calibrate the extrinsic parameters for the four cameras based on the algorithm proposed by Zhang et al [12] and Wu et al [11]. Fig. 2(c) demonstrates four point-cloud patches of the draped fabric captured by each camera. They were fused to generate a complete point cloud, as shown in Fig. 2(d). The corresponding triangulated mesh was generated after surface reconstruction, as shown in Fig. 2(e).

\subsection{Encoding WKS}

When fabric draping, there are different displacements for each vertex on the surface of the fabric. This deformation caused by the displacement is one of the manifestations of the comprehensive performance of the fabric. So far, the analysis of drape configuration has remained at the number of drape nodes, the unevenness of angles between drape peaks, the unevenness of the wavelength, etc [13]. However, in the computer graphics field, the shape analysis of 3D models has emerged a variety of shape descriptors, among which are well-known WKS, heat kernel signature (HKS) [14] and 3D scale-invariant feature transform (SIFT) features. These theoretical studies have brought 


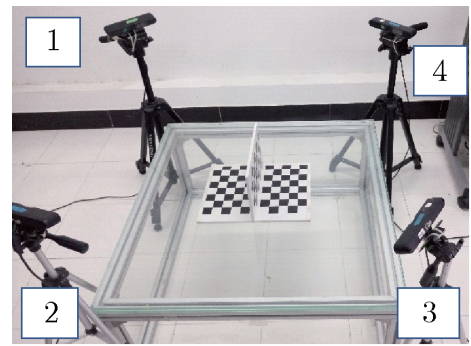

(a)

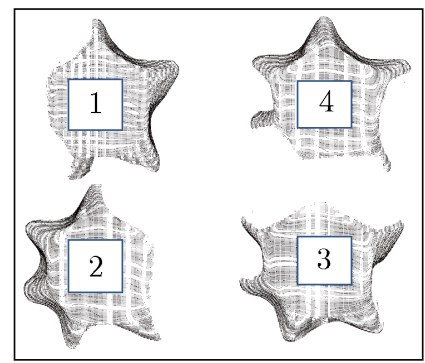

(c)

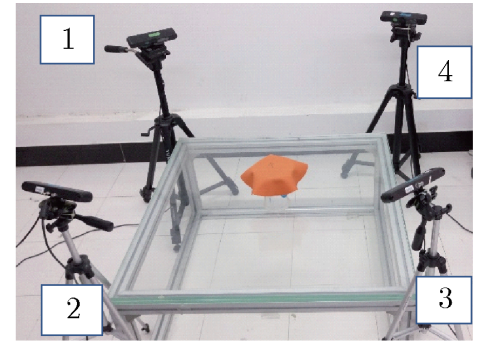

(b)

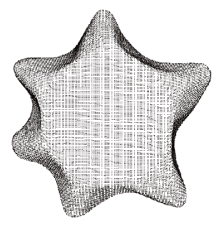

(d)

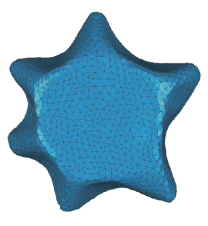

(e)

Fig. 2: The workflow of scanning a draped fabric (a) Calibration. (b) scanning. (c) four patches (d) completed 3D points cloud. (e) 3D triangulated mesh

great convenience to shape analysis.

However, so far, there are no 3D shape descriptors used for the analysis of fabric drape. Mathieu Aubry et al proved that WKS is more effective in analyzing three-dimensional shapes than other kinds of shape descriptors such as Fourier descriptors and HKS. Therefore, the WKS of fabric model was used to match the fabric in this study. The WKS was proposed by Aubry et al. in 2011. They introduced quantum mechanics and energy equations to the 3D surface. Aubry et al used the cotangent theorem to calculate the Laplacian-Beltramy (LB) operator of the model. The model is transformed into the spectral space by decomposing the LB operator. Then, in the spectral space, the function value of the energy equation is calculated by using the eigenvalues and eigenvectors. The function value is WKS, which reflects the quantum energy distribution in different energy levels.

For triangular mesh, the wave kernel function (energy function) of each vertex with respect to time is the solution of the wave Equation (1):

$$
\left(i \Delta+\frac{\partial}{\partial_{t}}\right) \psi(x, t)=0
$$

where, $i$ is the quantum particle parameter, $\Delta$ denotes the Laplacian-Beltramy operator on the surface, and $\psi(x, t)$ is the wave kernel function. The wave kernel function (energy function) can be expressed as Equation (2):

$$
\psi_{E}(x, t)=\sum_{k=0}^{\infty} e^{i v_{k} t} \emptyset_{k}(x) f_{E}\left(v_{k}\right)
$$

where, $\psi E(x, t)$ is the residual energy of the quantum particles corresponding to the vertex $x$, as well as the probability distribution of the quantum particles at this level. Since the frequency 
is closely related to the energy of the quantum particles, $v_{k}$ is the frequency information corresponding to the energy of the particle. $\varnothing(x)$ is an eigenvector function corresponding to the frequency information. $f_{E}\left(v_{k}\right)$ is the frequency probability distribution measured at vertex $x$ when the initial energy is $E$. According to Equation (2), the wavelet kernel signature is defined as the average probability that the energy of the quantum particle is $E$ at vertex $x$. Therefore, Equation (3) is defined,

$$
\operatorname{WKS}(E, x)=\lim _{T \rightarrow \infty} \frac{1}{T} \int_{0}^{T}\left|\psi_{E}(x, t)\right|^{2}
$$

Since the functions of $e^{-i E_{k} t}$ are orthogonal for the L2 norm, WKS can be expressed as Equation $(4)$

$$
\operatorname{WKS}(E, x)=\sum_{k=0}^{\infty} \varnothing_{k}^{2}(x) f_{E}^{2}\left(E_{k}\right)
$$

The time parameter of the wavelet kernel signature is replaced by an initial energy compared to the descriptor with time parameters. Eliminating the influence of time parameters on the eigenvector greatly increases the speed of the computation. $\varnothing(x)$ is an eigenvector function corresponding to the frequency information. $f_{E}\left(E_{k}\right)$ is the energy probability distribution at vertex $x$ with respect to the initial energy $E_{k}$. Here the energy probability distribution is a measure of the frequency probability distribution. The above Equation is the theoretical study of WKS. In practice, the WKS feature is defined as Equation (5).

$$
\operatorname{WKS}(x, E)=C_{E} \sum_{k} \varnothing_{k}^{2}(x) e^{\frac{-\left(E-\log E_{k}\right)^{2}}{2 \sigma^{2}}}
$$

where, $C_{E}=\left(\sum_{k} e^{\frac{-\left(E-\log E_{k}\right)^{2}}{2 \sigma^{2}}}\right)^{-1}$. E is the initial energy, which is closely related to the eigenvalues of the LB operator. $\varnothing(x)$ is the eigenvector of the LB operator. $K$ represents the $K^{\text {th }}$ the eigenvalue of the operator. $E_{k}$ is the initial energy corresponding to the $K^{t h}$ eigenvalue. $\sigma$ was variance.

In our experiments, the number of eigenvalues of Laplacian, marked as $M$, was set to 300 . The scales of $E$, marked as $N$, was set to 100 . The minimum value of $E, E_{\min }$ is set to $\log \left(E_{1}\right)+2 \sigma$, The maximum of $E, E_{\max }$ is set to $\log \left(E_{N}\right)-2 \sigma$. The increment $\delta$ in $E$ is set to $\left.E_{\max }-E_{\min }\right) / M$. The variance $\sigma$ was set to $7 \delta$. The algorithm used in this study was the same as Mathieu Aubry's research [15].

For the circular fabric sample, the displacement of vertices on the boundary is bigger than the interior of the fabric contrast. This means that studying on the boundary of the fabric can not only reduce the amount of computation but also refine the feature. The most important is that the WKS can reflect the local feature of each vertex. In another aspect, WKS of each vertex is a high dimensional signature. It is difficult to evaluate the similarity between fabrics by comparing multiple WKSs. Inspired by the model of bag-of-words [16], we proposed to encode WKSs of vertices on the $3 \mathrm{D}$ boundary into a vector. The WKS described below in this study refers to the WKSs of vertices on the boundary. The method of encoding WKS into vector was described in Fig. 3. 

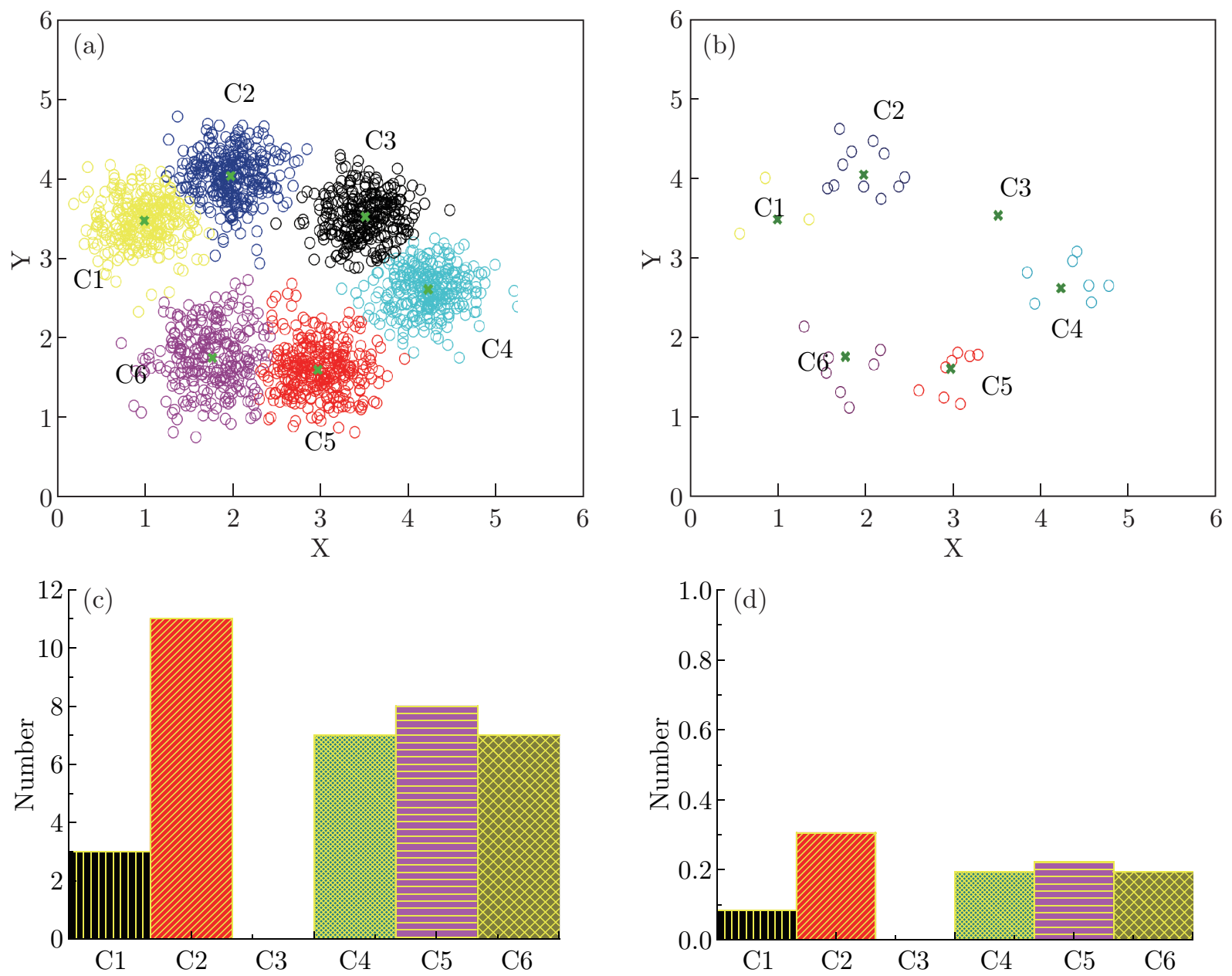

Fig. 3: The schematic diagram of encoding WKS into a vector ((a) K-Means result of WKS of all fabric samples; (b) Classification of WKS for single fabric sample; (c) Counting the number of each center; d-Normalizing the vector)

As shown in Fig. 3, the WKSs of all specimens were supposed to be clustered into six clusters with K-Means. Six cluster centers $\left(\left\{C_{1}, C_{2}, \cdots, C_{6}\right\}\right)$ were obtained. Classify the WKSs of an unknown specimen with the six cluster centers, as shown in Fig. 3(b). Count the number of WKSs belong to each cluster, as shown in Fig. 3(c). Normalize the result of Fig. 3(c) to make the sum of all components equal to 1, as shown in Fig. 3(d).

In this study, the number of eigenvalues of WKS is 300. The number of energy scales of WKS is 100. The number of cluster centers is set eighteen experimentally. The corresponding cluster centers are marked as $\left\{C_{1}, C_{2}, \cdots, C_{18}\right\}$. Each cluster center is also a vector of length 100 .

\subsection{Matching Fabrics Based on WKS and DR}

To match fabrics accurately, the encoded WKS, i.e., $\left\{C_{1}, C_{2}, \cdots, C_{18}\right\}$ was combined with DR. The new combined feature is $\left\{C_{1}, C_{2}, \cdots, C_{18}, D R\right\}$. The method based on the new feature is completed by calculating the Euclidean distance between the new features. The smaller the distance between the features, the better the matching results of the fabric to be matched. 


\subsection{Matching Fabrics with a Vector Consisting of Drape Indicators}

The drape coefficient $(D R)$ of the fabric is an indicator to characterize the ability of a fabric to drape. The number of fabric drape nodes $(N)$ can characterize the morphology of the draped fabric, as well as the solid rate $\left(R_{s}\right)$ of fabric drape [13]. Therefore, the contrast experiment uses a vector consisting of the three drape indicators: the drape coefficient, the number of fabric drape nodes, and the solid value of the drape fabric. As shown in Fig. $4, S_{1}$ refers to the area surrounded by the red curve. $S_{2}$ refers to the area surrounded by the minimal bounding circle. $R_{s}$ refers to the ratio of $S_{1}$ to $S_{2}$. With removing dimension, the vector was used as a basis for fabric matching. The matching method for the contrast experiment is completed by calculating the Euclidean distance between the vectors. The smaller the distance between the indicator vectors, the better the matching results of the fabric to be matched.

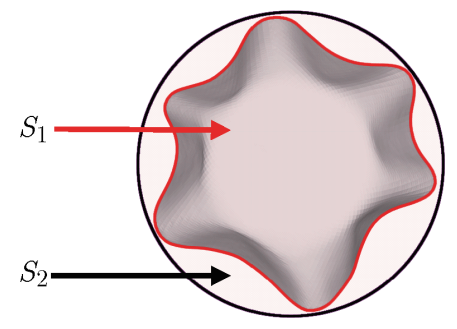

Fig. 4: The top view image of a draped fabric and the minimum bounding circle

\subsection{Computing the Similarity of Fabrics Based on KES-FB Parame- ters}

With various factors affecting the mechanical properties and structural parameters of fabric, Wu et al [17] compared the similarity of fabrics with a priority matrix of fuzzy similarity. Inspired by their work, we computed the similarity of fabrics by constructing a fuzzy similarity matrix. Wang et al [18] comprehensively compared the existing 13 methods of constructing a fuzzy similarity matrix. They proved that the absolute reciprocal method is most suitable for constructing fuzzy similar matrices. Therefore, absolute value reciprocal was used to construct the fuzzy similarity matrix $R$ in this study.

$$
\begin{aligned}
& R=\left\{\begin{array}{cccc}
r_{11} & r_{12} & \cdots & r_{1 n} \\
r_{21} & r_{22} & \cdots & r_{2 n} \\
\vdots & \vdots & \ddots & \vdots \\
r_{n 1} & r_{n 2} & \cdots & r_{n n}
\end{array}\right\} \\
& r_{i j}= \begin{cases}1 & i=j \\
\frac{c}{\sum_{k=1}^{m}\left|x_{i k}-x_{j k}\right|} & i \neq j\end{cases}
\end{aligned}
$$

where, $r_{i j}$ is the similarity of sample $i$ and sample $j$. The larger the value of $r_{i j}$, the greater the similarity of sample $i$ and sample $j$. $c$ is a constant. $m$ refers to the length of the feature. $x_{i k}$, $x_{j k}$ are the $k^{t h}$ feature components of samples $i$ and $j$ respectively. 
In this study, fabric weight, average bending stiffness, average bending hysteresis moment, average shearing stiffness, and average shearing hysteresis moment of the fifty-one fabrics was measured with KES-FB. The fuzzy similarity matrix $R$ is constructed with the measured five indicators as the characteristic components. Elements in each row of $R$ are sorted from largest to smallest. The larger the value of $r_{i j}$, the greater the similarity of sample $i$ and sample $j$. The matching results of Section 2.4 and Section 2.5 were evaluated with the ranking results.

\section{Results and Discussion}

The top view image of the draped fabric and four WKS curves of vertices on the 3D boundary are shown in Fig. 5.
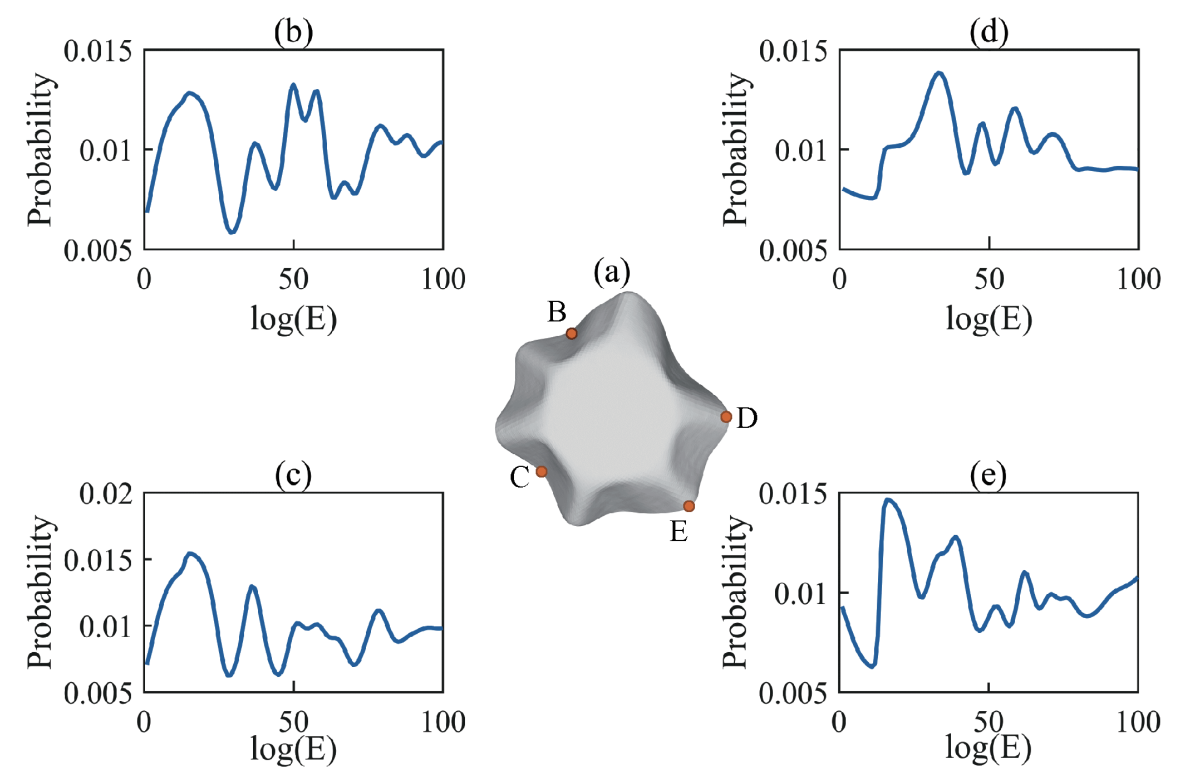

Fig. 5: Draped fabric and Wave Kernel Signatures (a-The top view image of a draped fabric; b-The WKS of vertex B; c-The WKS of vertex C; d-The WKS of vertex D; e-The WKS of vertex E)

According to the Schrodinger equation, each vertex on the triangle surface is associated with a Wave Kernel Signature. As shown in Fig. 5, two vertices on the left are the drape troughs. The difference between them is the local deformation. Two vertices on the right side are located at the drape peaks. There is an obvious difference between Fig. 5(b) and Fig. 5(d). The similarity between Fig. 5(b) and Fig. 5(c) is comparatively high. This verified that WKS is suitable to distinguish the local deformation of $3 \mathrm{D}$ objects.

The matching results of fabric based on vectors consisting of drape indicators and WKS+DR are shown in Fig. 6. Since a large number of samples, only the fabrics with obvious different matching results are shown in this section. As shown in Fig. 6, the 'Input' column represents the top view image of the given fabrics. The 'Vector' column represents the matching results with the method based on vectors consisting of drape indicators. The WKS+DR column represents the matching results with the method based on WKS+DR.

As shown in Fig. 6, the matching results ('Vector' column) with the method of vectors consisting of drape indicators are different in drape configuration with the matching fabrics ('Input' column). 


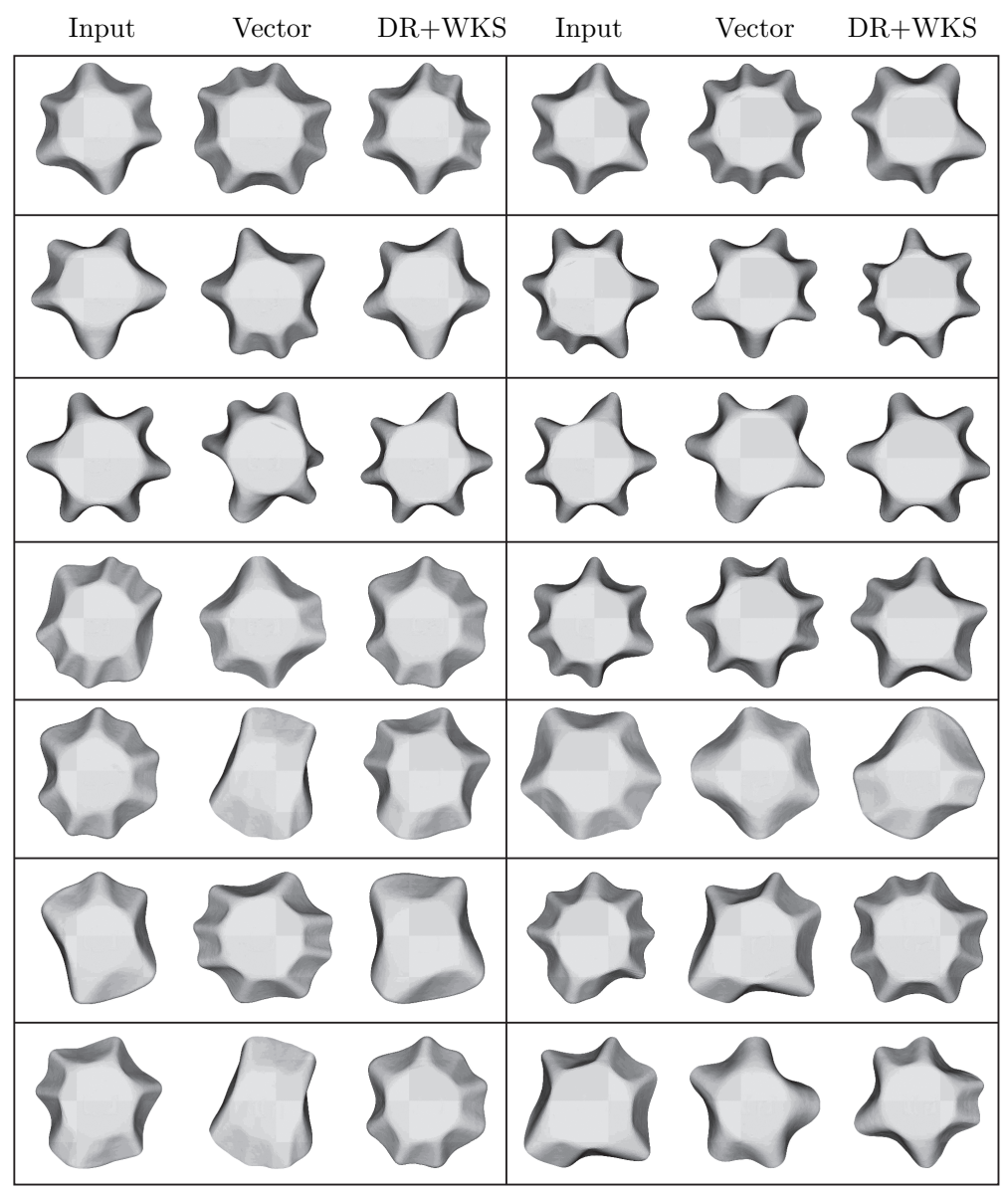

Fig. 6: The result of fabric matching based on vectors and WKS+DR

The difference includes anisotropy, local deformation, number of drape nodes, the unevenness of wavelength and the unevenness of angles between drape peaks. The most obvious property is the local deformation of the triangle surface. However, the matching results ('DR+WKS' column) with the method of DR+WKS are quite similar to the matching fabrics ('Input' column). Especially, the local deformation matches well. This phenomenon can be explained as the drape performance of fabric dependence on the structural parameters and mechanical properties of the fabric. With a hierarchical structure, the drape results of the same fabric may be discrete. Similar fabrics may have the same 3D drape results. WKS could distinguish 3D shapes and the drape coefficient could evaluate the drape-ability of fabric. This makes that the combination of WKS and DR could match fabric contrast subjectively. According to the evaluation criteria for fabric matching proposed in Section 2.6, the accuracy of the matching results based on DR is $78.9 \%$. The accuracy of the matching based on the combination of WKS and DR is $89.6 \%$.

\section{Conclusions}

In this study, the 3D point clouds of draped fabrics were scanned via a self-built 3D scanning device followed with triangulation. The drape coefficient, the number of fabric drape nodes, and the solid rate of a draped fabric were extracted, as well as the WKS of vertices on the draped fabric. The WKS of points on the 3D boundary was encoded into a vector of length eighteen. The 
encoded WKS and a vector consisting of three drape indicators were used to match the fabric. It is concluded that WKS could distinguish the local deformation of draped fabrics. The method based on DR+WKS is more subjective than the vector consisting of drape indicators in matching fabrics. The accuracy of this method could reach $89.6 \%$. The fabrics obtained with this method have high similarity with the matching fabric in both drape-ability and local deformation of the triangular mesh. The study could provide significant guidance for fabric matching.

\section{Acknowledgment}

This work was supported by the National Natural Science Foundation of China (Grant No. 61572124) and the Special Excellent Ph.D. International Visit Program by Donghua University (DHU).

\section{References}

[1] Jeong Y J, Phillips D G. A Study of Fabric-drape Behavior with Image Analysis. Part II: The Effects of Fabric Structure and Mechanical Properties on Fabric Drape, The Journal of Textile Institute, 1998; 89(1): 70-79.

[2] Xue Z B, Zeng X Y, Koehl L, Chen Y. Extracting fabric hand information from visual representations of flared skirts. Textile Research Journal. 2014; 84(3): 246-266.

[3] Kuijpers A. 2017. Evaluation of physical and virtual fabric drape created from objective fabric properties. Master diss. The University of Manchester, UK.

[4] Wang S B, Zhou H, Shen Y, Liu X M. Three-Dimensional Shape Reconstruction Algorithm of Fabric Drape-ability Based on Raster Imaging. Journal of engineering graphics. 2008; 29: 136-141.

[5] Mah T, Song G W. An investigation of the assessment of fabric drape using three-dimensional body scanning. Journal of the Textile Institute Proceedings \& Abstracts. 2010; 101: 324-335.

[6] $\mathrm{Hu} \mathrm{K}, \mathrm{Wu}, \mathrm{G}$ Zhong Y Q. Reconstruction and Measurement of Fabric Drape Based on Image Sequences. Journal of Donghua University. 2016; 42: 674-698.

[7] Niwa M, Seto F. Relationship between Drapability and Mechanical Properties of Fabrics. Journal of the Textile Machinery Society of Japan. 1998; 51 (9): 499-506.

[8] Narahari K, Lamar T M, Pradeep P, Jeffrey E. Enhancing accuracy of drape simulation. Part I: Investigation of drape variability via 3D scanning. Journal of the Textile Institute Proceedings \& Abstracts. 2008; 99: 211-219.

[9] Mathieu Aubry, Ulrich Schlickewei and Daniel Cremers. The Wave Kernel Signature: A Quantum Mechanical Approach to Shape Analysi. IEEE International Conference on Computer Vision Workshops. 2011: 1626-1633.

[10] Yu Z C, Zhong Y Q, Gong R, et al. Fabric Inquiry Based on Wavelet Kernel Signature and Drape Coefficient. Textile Bioengineering and Informatics Society. 2019, 295-303.

[11] Wu G. 2017. Three-Dimensional reconstruction and shape analysis of human feet based on multiple depth cameras. PhD diss. Donghua University, China.

[12] Zhang Z Y. A Flexible New Technique for Camera Calibration. IEEE Computer Society, 2000; 22: 1330-1334.

[13] E Carrera-Gallissà, X Capdevila, J Valldeperas. Evaluating drape shape in woven fabrics, J Text Inst, vol. 108, no. 3, 2016, 325-336. 
[14] Sun J, Ovsjanikov M, Guibas L. A Concise and Provably Informative Multi-Scale Signature-Based on Heat Diffusion. Eurographics Symposium on Geometry Processing. 2009; 28(5): 1383-1392.

[15] Aubry M, Schlickewei U, Cremers D. The Wave Kernel Signature: A Quantum Mechanical Approach to Shape Analysi. IEEE International Conference on Computer Vision Workshops. 2011: 1626-1633.

[16] Peng X J, Wang L M, Wang X X, Qiao Y. Bag of visual words and fusion methods for action recognition: Comprehensive study and good practice. Computer Vision and Image Understanding. 2016; 150: 109-125.

[17] Wu S M, Wang X H. Fuzzy Mathematical Application for Fabrics Drape Analysis. Journal of Yili Normal University (Natural Science Edition). 2014; 8(1): 15-18.

[18] Wang X Z, Shu H C. Construction of Fuzzy Similar Matrix. Journal of Jishou University (Natural Science Edition). 2004; 24(3): 37-41. 